See discussions, stats, and author profiles for this publication at: https://www.researchgate.net/publication/339439443

\title{
Conversational Agents for Health and Wellbeing
}

Conference Paper · April 2020

DOI: $10.1145 / 3334480.3375154$

CITATIONS

0

10 authors, including:

A. Baki Kocaballi

University of Technology Sydney

43 PUBLICATIONS 239 CITATIONS

SEE PROFILE

Some of the authors of this publication are also working on these related projects:

Conversational Agents in Healthcare View project

Conversational Al for Persuasion and Behavior Change View project
READS

291

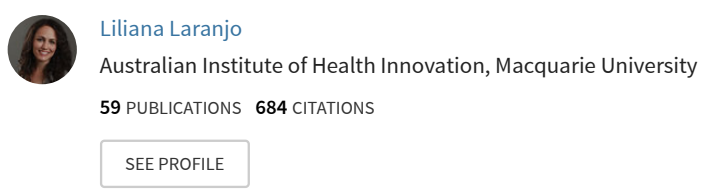




\section{Conversational Agents for Health and Wellbeing}

\begin{tabular}{|c|c|}
\hline A. Baki Kocaballi & Q. Vera Liao \\
\hline Juan C. Quiroz & IBM Research AI \\
\hline Liliana Laranjo & New York, USA \\
\hline Dana Rezazadegan & vera.liao@ibm.com \\
\hline \multicolumn{2}{|l|}{ Macquarie University } \\
\hline NSW, Australia & Sun Young Park \\
\hline \{baki.kocaballi; juan.quiroz; & University of Michigan \\
\hline liliana.Iaranjo; & Michigan, USA \\
\hline dana.rezazadegan\}@mq.edu.au & sunypark@umich.edu \\
\hline Rafal Kocielnik & Robert J. Moore \\
\hline University of Washington & IBM Research \\
\hline $\begin{array}{l}\text { Washington, USA } \\
\text { rafal.kocielnik@gmail.com }\end{array}$ & $\begin{array}{l}\text { California, USA } \\
\text { rjmoore@us.ibm.com }\end{array}$ \\
\hline Leigh Clark & Adam Miner \\
\hline Swansea University & Stanford University \\
\hline I.m.h.clark@swansea.ac.uk & $\begin{array}{l}\text { California, USA } \\
\text { aminer@stanford.edu }\end{array}$ \\
\hline
\end{tabular}

Permission to make digital or hard copies of part or all of this work for personal or classroom use is granted without fee provided that copies are not made or distributed for profit or commercial advantage and that copies bear this notice and the full citation on the first page. Copyrights for third-party components of this work must be honored. For all other uses, contact the Owner/Author.

CHI'20 Extended Abstracts, April 25-30, 2020, Honolulu, HI, USA

CHI'20 Extended Abstracts, April 25-30, 2020, Honol
(C) 2020 Copyright is held by the owner/author(s).

(C) 2020 Copyright is held by the owner/a

ACM ISBN 978-1-4503-6819-3/20/04.
DOI: https://doi.org/10.1145/3334480.3375154

\begin{abstract}
Conversational agents have increasingly been deployed in healthcare applications. However, significant challenges remain in developing this technology. Recent research in this area has highlighted that: i) patient safety was rarely evaluated; ii) health outcomes were poorly measured, and iii) no standardised evaluation methods were employed. The conversational agents in healthcare are lagging behind the

developments in other domains. This one-day workshop aims to create a roadmap for healthcare conversational agents to develop standardised design and evaluation frameworks. This will prioritise health outcomes and patient safety while ensuring a high-quality user experience. In doing so, this workshop will bring together researchers and practitioners from $\mathrm{HCI}$, healthcare and related speech and chatbot domains to collaborate on these key challenges.
\end{abstract}

\section{Author Keywords}

Conversational agent; voice interface; speech interface, chatbots; healthcare; health informatics.

\section{CSS Concepts}

- Human-centered computing $\rightarrow$ Human computer interaction $(\mathrm{HCI})$ 


\section{Background}

Conversational agents are systems that engage in conversations with humans using text or spoken language. Advances in speech recognition, natural language processing, and machine learning have led to an increasing adoption and use of conversational agents. Despite the varied terminology $[2,18]$, there are numerous commonly used technologies featuring conversational interfaces: chatbots, which have the ability to engage in "small talk" and casual conversation; embodied conversational agents, which involve a computer-generated character (e.g. avatar, virtual agent) simulating face-to-face conversation with verbal and nonverbal behavior; and intelligent assistants such as Apple Siri and Google Assistant.

Over the last two decades, there is a rapidly growing market of agents for health-related tasks, with Alexa alone having over 1000 "skills" in the health category. Research has shown the potential benefits of using conversational agents in healthcare $[2,17]$. Several randomized controlled trials of interventions involving embodied conversational agents have shown significant improvements in physical activity, dietary, and accessibility, among other outcomes [5, 6, 10, 30]. Although, there has been an increasing number of conversational agents using speech interfaces in $\mathrm{HCI}$ [8] with their benefits in accessibility [15, 27], the majority of the healthcare agents lack the capability to understand natural language speech input, allowing only for constrained user input such as multiple-choice of utterance options. Currently, using unconstrained natural language input for receiving medical advice is not recommended due to the risks involved [3].
In various healthcare settings, conversational agents play increasingly important roles such as assisting clinicians during the consultation [12], supporting consumers in changing their behaviors [16, 29], or assisting patients and elderly individuals in their living environments $[25,31]$. These opportunities also come with potential safety issues $[4,14,20]$ and

psychological and behavioral ramifications for the user $[11,19]$. A recent systematic review focusing on conversational agents in healthcare found that i) patient safety was rarely evaluated; ii) health outcomes were poorly measured, and iii) no standardised evaluation methods were employed [17]. Another review study focusing on personalization of healthcare conversational agents found that most of the studies implemented the personalization features without a theoretical or evidence-based support [13].

Because of the fundamental differences between audio and visual modalities, conversational interfaces have required us to rethink the application of design and evaluation factors well-established in graphical user interfaces such as affordances, constraints, feedback, and visibility. Although there have been some recent heuristic guidelines offered for human-AI interaction [1] and speech interfaces [24], there is not even a shared technical definition of what constitutes a "conversation" versus other kinds of natural-language-based interactions [22]. Many design questions need to be revisited for conversational interfaces such as: What are the best ways of navigation? What are the best ways to provide feedback or confirmation? How should the system deal with troubles in understanding? What constitutes a good user experience? What are people's perceptions and mental models about conversational agents? How do users build trust toward conversational 
agents? All these questions are more critical in the healthcare domain where user-system interactions may have serious unexpected or unintended consequences and risks.

More research studies are needed to understand the complexities of conversational interactions between patients, healthcare professionals, and technologies in various healthcare settings. For example, a new class of conversational technology referred to as the Digital Scribe aims to work with doctors as digital assistants in clinical encounters [9]. There are various challenges involved in introducing such conversational technologies in clinical settings such as automation bias, data ownership and privacy, professional autonomy, and medico-legal issues, going beyond the scopes of many design and evaluation methods [9] Therefore, in addition to more standardized design and evaluation methods to manage sensitive and risky situations with no harm, some ethical and regulatory factors and their implications need to be addressed.

Recent workshops at $\mathrm{CHI}$ and $\mathrm{CSCW}$ have examined the challenges and opportunities of conversational user interfaces in general $[7,23,28]$ and also looked into the use of conversational agents for cooperative work [26]. This workshop will focus on the design and evaluation of conversational agents in healthcare, which has unique needs related to safety, trust, and data sharing [21]. The aim is to create a roadmap to develop standardised design and evaluation frameworks prioritising health outcomes and patient safety while maintaining satisfactory user experience.

\section{Organizers}

A. Baki Kocaballi is a research fellow at the Centre for Health Informatics at Macquarie University. He has a $\mathrm{PhD}$ in Interaction Design and MSc in Information Systems. His research investigates the opportunities and challenges of designing and evaluating AI-enabled conversational agents in healthcare.

Juan C Quiroz is a research fellow at the Centre for Health Informatics at Macquarie University. He has a $\mathrm{PhD}$ in Computer Science, and his current research explores the natural language processing for the automated summarization of medical conversations through speech interfaces.

Liliana Laranjo is a medical doctor with a Master of Public Health from Harvard University, and a PhD in Health informatics. Liliana works at the Centre for Health Informatics at Macquarie University. Her current research focuses on person-centered health informatics, online social networks, artificial intelligence, and behavior change informatics.

Dana Rezazadegan is a post-doctoral research fellow at Macquarie University. She is currently working on using artificial intelligence to automate documentation and diagnosis for helping doctors in examination room. She studied her Ph.D. in Robotics and Autonomous systems with a focus on robotic vision and deep learning at Queensland University of Technology.

Rafał Kocielnik is a PhD student at Human Centered Design \& Engineering Department at University of Washington. His current research focuses on creating smart technologies for persuasion and behavior change through conversational interfaces. He also investigates 
broader user perceptions of AI systems and the ways of augmenting the design process with AI technologies.

Leigh Clark is a Lecturer in Computer Science at Swansea University. His research examines user interactions with speech interfaces, how design choices and contexts impact user perceptions and behaviors and how linguistic theories can be implemented and redefined in speech-based $\mathrm{HCI}$.

Vera Liao is a research staff member in IBM Research AI at T.J Watson Research Center. She has a PhD in $\mathrm{HCI}$ and master's degree in human factors from the University of Illinois at Urbana-Champaign. Her current research focuses on human-AI interactions, in particular the design of conversational agents and tools that support the development of agents.

Sun Young Park is an assistant professor at the University of Michigan in the Stamps School of Art and Design and the School of Information. Her research uses design ethnography to study patient engagement, patient-provider collaboration, patient-centered health technology, and technology adaptation. Her work has been awarded by the National Science Foundation (NSF) and the Agency for Healthcare Research and Quality (AHRQ)

Robert J. Moore is a scientist at IBM ResearchAlmaden. His current work applies conversation science to the design of conversational agents. His recent book outlines a methodology for conversational UX design. Bob has a Ph.D. in sociology with a concentration in Conversation Analysis.
Adam Miner is a licensed clinical psychologist, and instructor at Stanford University School of Medicine. He uses experimental and observational studies to improve the ability of conversational AI to recognize and respond to health issues. His current focus is the use of modern computational approaches, such as natural language processing, and AI to understand language patterns in psychotherapy.

\section{Website}

A workshop website to provide the details of the workshop and the accepted position papers will be online at: http://casforhealth.org

\section{Themes and Goals}

Conversational agents in healthcare is an emerging field of research with the potential to benefit health across a wide range of application domains. This workshop aims to address current issues and potential benefits by bringing together conversational UX designers, medical informatics, HCI, machine learning, and design researchers investigating:

\section{Studies and Cases}

- Use of conversational agents in clinical and community settings

- Experiences with existing conversational agents or research prototypes used in clinical settings by healthcare professionals

- Use of conversational agents for sensitive and mental health-related topics

- $\quad$ Patient experience, perception, and perspectives focusing on empowerment, safety, trust, mental models and etc. 


\section{Important Dates}

11 Dec 2019: Call for Participation released.

11 Feb 2020: Position papers deadline.

28 Feb 2020: Notification of acceptance.
Design, Development and Evaluation

- Emerging uses of artificial intelligence and machine learning methods in areas such as dialog management, spoken language understanding and response generation

- Novel applications of UX design and evaluation methods, and use and development of standardized measurement scales and tools

- Context-specific design guidelines to support the interactions between conversational agents and patients/providers

- $\quad$ Ethical and regulatory dimensions Understanding of design factors such as feedback, feedforward, and affordances within the context of conversational agents

- Evolving relationships, roles, and tasks for clinicians, patients, and caregivers with the introduction of conversational systems

- Design and development practices specific to the healthcare domain such as safety-critical dialogue, ML for healthcare, health intervention.

This workshop contributes to the growing body of knowledge on conversational agents. Participants will gain a better understanding of the different

characteristics, applications, design and evaluation methods of current conversational agents in healthcare. Given the growing popularity of conversational agents, and their increasing use in healthcare, it is crucial to develop a roadmap for researching more standardized design and evaluation frameworks prioritising health outcomes and patient safety while maintaining highquality user experience.

\section{Pre-workshop Plans}

The workshop aims to bring together researchers and practitioners from HCI, healthcare, medical informatics, conversational UX and interaction design, machine learning, and speech-based domains with an interest in conversational technologies to support health outcomes. The call for participation will be distributed across $\mathrm{HCI}, \mathrm{AI}$, design and health informatics mailing lists. The organizers will also use social networking, such as Twitter, to target a wider research audience. The organizers will also ask for submissions from personal contacts with relevant research expertise, from the organizers' home institutions, from contacts in the relevant industry and other non-academic institutions.

\section{Workshop Structure}

09:00 Arrival

09:15 Introduction

- Brief intro about the workshop scope, aims, and format

- A short roundtable discussion to introduce ourselves to each other

- Presentation and discussion of the outcomes of recent systematic reviews

\section{0:30 Coffee break}

\section{1:00 Session 1}

- $\quad$ Paper presentations by the workshop participants

- Writing affinity notes about the presentations

\section{2:00 Lunch break}

13:00 Session 2

- Interactive evaluation of some healthcare scenarios involving fictive 
conversational systems accompanied with affinity note-taking

\section{4:00 Coffee break}

\section{4:15 Session 3}

- Generating an affinity diagram

- Generating a map of actors, settings, technologies, design and evaluation factors

\section{4:45 Wrap up}

- $\quad$ Summarizing the day and discussing the next steps with the participants

\section{5:00 End}

\section{Post-workshop Plans}

After the workshop, we will organize a special issue either in an $\mathrm{HCI}$ or health informatics journal, addressing the research themes of the workshop. In addition, the workshop contributors will be invited to work on a joint journal paper outlining a roadmap for design and evaluation challenges associated with conversational agents in healthcare.

\section{Call for Participation}

This one-day workshop at the CHI 2020 in Hawaii, USA invites HCI designers, researchers, healthcare professionals, health informatics researchers, and AI developers and researchers to contribute to the emerging area of conversational agents in healthcare.

The aim of this workshop is to: i) identify challenges, opportunities, and research issues in developing more standardized design and evaluation frameworks for conversational systems, ii) discuss ongoing studies employing conversational interfaces to improve health outcomes, iii) review natural language input and output technologies playing a key role in shaping conversational experiences, and iv) generate a map of actors, settings, technologies, design and evaluation factors to guide future research in this emerging area. We invite researchers and professionals to share their related work and discuss future research directions. We would accept position papers including but not limited to the following topics:

- Use of conversational agents in clinical and community settings

- Preliminary results on ongoing empirical studies of healthcare conversational agents

- Conversational agent studies focusing on supporting patient-centeredness, patient empowerment, and patient safety or professional autonomy

- $\quad$ Risks and biases associated with design and use of conversational technologies

- Emerging uses of AI and ML methods in areas such as dialog management, speech summarization or error recovery

- Novel applications of UX design and evaluation methods.

Position papers should be 2-4 pages long (excluding references) in the CHI EA format. There will be an internal peer-review process between the authors of the submitted papers. Papers will be selected based on relevance to the workshop, quality of the contribution, and ability to contribute to discussion. Submissions and questions should be emailed to casforhealth@gmail.com. At least one author of each accepted paper must attend the workshop. 


\section{Acknowledgements}

This workshop is supported by the National Health and Medical Research Council (NHMRC) grant APP1134919 (Centre for Research Excellence in Digital Health) and Programme Grant APP1054146 as part of the Digital Scribe Project led by Professor Enrico Coiera. This work was also supported by grants from the National Institutes of Health, National Center for Advancing Translational Science, Clinical and Translational Science Award (KL2TR001083 and UL1TR001085), the Stanford Department of Psychiatry Innovator Grant Program, and the Stanford Human-Centered AI Institute. We would like to thank Prof Michael McTear for his comments on an earlier draft of this proposal.

\section{References}

[1] Saleema Amershi, Dan Weld, Mihaela Vorvoreanu, Adam Fourney, Besmira Nushi, Penny Collisson, Jina Suh, Shamsi Iqbal, Paul N. Bennett, Kori Inkpen, Jaime Teevan, Ruth Kikin-Gil and Eric Horvitz. 2019. Guidelines for Human-AI Interaction. 2019. Guidelines for Human-AI Interaction. In Proc. of CHI.

[2] T. Bickmore and T. Giorgino. 2006. Health Dialog Systems for Patients and Consumers. Journal of biomedical informatics 39, 5, 556-571.

[3] T. W. Bickmore, H. Trinh, S. Olafsson, T. K. O'Leary, R. Asadi, N. M. Rickles and R. Cruz. 2018. Patient and Consumer Safety Risks When Using Conversational Assistants for Medical Information: An Observational Study of Siri, Alexa, and Google Assistant. J Med Internet Res 20, 9, e11510.

[4] Timothy Bickmore, Ha Trinh, Reza Asadi and Stefan Olafsson. 2018. Safety First: Conversational Agents for Health Care. In Studies in Conversational UX Design, Robert J. Moore et al. Eds. Springer International Publishing, Cham, 33-57.

[5] Timothy W. Bickmore, Daniel Schulman and Candace Sidner. 2013. Automated Interventions for Multiple Health Behaviors Using Conversational Agents. Patient Educ Couns 92, 2, 142-148.

[6] Timothy W. Bickmore, Rebecca A. Silliman, Kerrie Nelson, Debbie M. Cheng, Michael Winter, Lori Henault and Michael K. Paasche-Orlow. 2013. A Randomized Controlled Trial of an Automated Exercise Coach for Older Adults. J Am Geriatr Soc 61, 10, 1676-1683.

[7] Leigh Clark, Benjamin R. Cowan, Justin Edwards, Cosmin Munteanu, Christine Murad, Matthew Aylett, Roger K. Moore, Jens Edlund, Eva Szekely, Patrick Healey, Naomi Harte, Ilaria Torre and Philip Doyle. 2019. Mapping Theoretical and Methodological
Perspectives for Understanding Speech Interface Interactions. In Proc. of Ext. Abstracts of $\mathrm{CHI}$.

[8] Leigh Clark, Philip Doyle, Diego Garaialde, Emer Gilmartin, Stephan Schlögl, Jens Edlund, Matthew Aylett, João Cabral, Cosmin Munteanu, Justin Edwards and Benjamin R Cowan. 2019. The State of Speech in Hci: Trends, Themes and Challenges. Interacting with Computers.

[9] Enrico Coiera, Baki Kocaballi, John Halamka and Liliana Laranjo. 2018. The Digital Scribe. npj Digital Medicine 1, 1, 58.

[10] Roger A. Edwards, Timothy Bickmore, Lucia Jenkins, Mary Foley and Justin Manjourides. 2013. Use of an Interactive Computer Agent to Support Breastfeeding. Matern Child Health J 17, 10, 1961-1968.

[11] Annabel Ho, Jeff Hancock and Adam S. Miner. 2018. Psychological, Relational, and Emotional Effects of Self-Disclosure after Conversations with a Chatbot. J Commun 68, 4, 712-733.

[12] Jeffrey G. Klann and Peter Szolovits. 2009. An Intelligent Listening Framework for Capturing Encounter Notes from a Doctor-Patient Dialog. BMC Med Inform Decis Mak 9, Suppl 1, S3.

[13] A. Baki Kocaballi, Shlomo Berkovsky, Juan C. Quiroz, Liliana Laranjo, Huong Ly Tong, Dana Rezazadegan, Agustina Briatore and Enrico Coiera. 2019. Personalization of Conversational Agents in Healthcare: A Systematic Review. J Med Internet Res.

[14] A. Baki Kocaballi, Juan C. Quiroz, Shlomo Berkovsky, Dana Rezazadegan, Farah Magrabi, Enrico Coiera and Liliana Laranjo. 2020. Responses of Conversational Agents to Health and Lifestyle Prompts: Investigation of Appropriateness and Presentation Structures. Journal of Medical Internet Research.

[15] Rafal Kocielnik, Elena Agapie, Alexander Argyle, Dennis T Hsieh, Kabir Yadav, Breena Taira and Gary 
Hsieh. 2019. Harborbot: A Chatbot for Social Needs Screening. In Proceedings of AMIA. Washington.

[16] Rafal Kocielnik, Lillian Xiao, Daniel Avrahami and Gary Hsieh. 2018. Reflection Companion: A Conversational System for Engaging Users in Reflection on Physical Activity. Proc. ACM Interact. Mob. Wearable Ubiquitous Technol. 2, 2, 1-26.

[17] Liliana Laranjo, Adam G. Dunn, Huong Ly Tong, A. Baki Kocaballi, Jessica Chen, Rabia Bashir, Didi Surian, Blanca Gallego, Farah Magrabi, Annie Y. S. Lau and Enrico Coiera. 2018. Conversational Agents in Healthcare: A Systematic Review. J Am Med Inform Assn 25, 9, 1248-1258.

[18] Michael McTear, Zoraida Callejas and David Griol. 2016. The Conversational Interface: Talking to Smart Devices. Springer

[19] Adam S. Miner, A. Milstein and J. T. Hancock. 2017. Talking to Machines About Personal Mental Health Problems. Jama 318, 13, 1217-1218.

[20] Adam S. Miner, A. Milstein, S. Schueller, R. Hegde, C. Mangurian and E. Linos. 2016. Smartphone-Based Conversational Agents and Responses to Questions About Mental Health, Interpersonal Violence, and Physical Health.JAMA internal medicine 176,5,619-25.

[21] Adam S Miner, Nigam Shah, Kim Bullock, Bruce Arnow, Jeremy Bailenson and Jeff Hancock. 2019. Key Considerations for Incorporating Conversationa Ai in Psychotherapy. Frontiers in Psychiatry 10, 746.

[22] Robert J. Moore and Raphael Arar. 2019. Conversational UX Design: A Practitioner's Guide to the Natural Conversation Framework. ACM.

[23] Robert J. Moore, Raphael Arar, Guang-Jie Ren and Margaret H. Szymanski. 2017. Conversational UX Design. In Proc. Extended Abstracts of CHI. ACM.

[24] Christine Murad, Cosmin Munteanu, Leigh Clark and Benjamin R. Cowan. 2018. Design Guidelines for
Hands-Free Speech Interaction. In Proc. of Mobile HCI'18 Adjunct. ACM.

25] Toyoaki Nishida, Atsushi Nakazawa, Yoshimasa Ohmoto and Yasser Mohammad. 2014.

Conversational Informatics: A Data-Intensive Approach with Emphasis on Nonverbal Communication (2014 edition). Springer, Tokyo.

[26] Martin Porcheron, Joel E. Fischer, Moira McGregor, Barry Brown, Ewa Luger, Heloisa Candello and Kenton O'Hara. 2017. Talking with Conversational Agents in Collaborative Action. In Proc. of CSCW Companion.

[27] Alisha Pradhan, Kanika Mehta and Leah Findlater. 2018. "Accessibility Came by Accident": Use of VoiceControlled Intelligent Personal Assistants by People with Disabilities. In Proc. of CHI'18. ACM

[28] Stuart Reeves, Martin Porcheron, Joel E. Fischer, Heloisa Candello, Donald McMillan, Moira McGregor, Robert J. Moore, Rein Sikveland, Alex S. Taylor, Julia Velkovska and Moustafa Zouinar. 2018. Voice-Based Conversational UX Studies and Design. In Proc. of Extended Abstracts of CHI. ACM.

[29] Marie A. Sillice, Patricia J. Morokoff, Ginette Ferszt, Timothy Bickmore, Beth C. Bock, Ryan Lantini and Wayne F. Velicer. 2018. Using Relational Agents to Promote Exercise and Sun Protection: Assessment of Participants' Experiences with Two Interventions. ] Med Internet Res 20, 2, e48.

[30] Alice Watson, Timothy Bickmore, Abby Cange, Amba Kulshreshtha and Joseph Kvedar. 2012. An InternetBased Virtual Coach to Promote Physical Activity Adherence in Overweight Adults: Randomized Controlled Trial. J Med Internet Res 14, 1, e1.

[31] Maria Klara Wolters, Fiona Kelly and Jonathan Kilgour. 2016. Designing a Spoken Dialogue Interface to an Intelligent Cognitive Assistant for People with Dementia. Health Informatics Journal 22, 4, 854-866. 